\title{
ANÁLISE DA CESSAÇÃO DO TABAGISMO DURANTE E APÓS O TRATAMENTO E
}

\author{
FATORES ASSOCIADOS
}

Ana Cláudia Garabeli Cavalli Kluthcovsky ${ }^{1}$
Cecília Fanha Dornelles $^{2}$
Erildo Vicente Müller $^{3}$
Allan Catarino Kiska Torrani ${ }^{4}$
Alisson Ferreira Pupulim $^{5}$
Carlos Rory Pucci Filho $^{6}$
Maki Caroline Nakamura
Matheo Augusto Morandi Stumpf

RESUMO: O tabagismo possui relação com diversas doenças e seu tratamento traz benefícios para fumantes ativos e passivos. $O$ objetivo desse estudo foi analisar os resultados do tratamento em pacientes tabagistas e possíveis fatores associados. Estudo retrospectivo com análise dos dados de 62 pacientes tratados em um projeto de extensão universitária. Foram coletados dados socioeconômicos, clínicos, referentes ao tabagismo e sobre os resultados da cessação do tabagismo durante o período de tratamento e após o tratamento (tempo médio de 8,0 $\pm 2,0$ meses após o início do tratamento). Para as análises estatísticas foram utilizados os testes Qui-quadrado e Exato de Fisher, com nível de significância de $5 \%$. Durante o período de tratamento $51,6 \%$ dos pacientes pararam de fumar e $37,1 \%$ reduziram o número de cigarros por dia. Dos 54 pacientes que responderam aos contatos telefônicos após o tratamento, 31,5\% permaneciam sem fumar, $27,8 \%$ estavam fumando número reduzido de cigarros por dia e $16,6 \%$ apresentaram recaída. Frequência de presenças aos encontros durante o tratamento inferior a 5 vezes $(p<0,001)$ e grau de dependência à nicotina elevado ou muito elevado $(p=0,03)$ foram associados a não parar de fumar durante o tratamento. Observou-se uma boa proporção de cessação do tabagismo durante e após o tratamento. Incentivo deve ser dado para que os pacientes compareçam aos encontros de tratamento, com atenção especial para aqueles com grau de dependência à nicotina elevado ou muito elevado.

Palavras-chave: Tabagismo. Abandono do uso do tabaco. Produtos para o abandono do uso de tabaco. Programa Nacional de Controle do Tabagismo.

\section{ANALYSIS OF SMOKE CESSATION DURING AND AFTER TREATMENT AND}

\footnotetext{
${ }^{1}$ Médica. Doutora em Medicina Interna e Ciências da Saúde. Universidade Estadual de Ponta Grossa. E-mail: anafabio2009@gmail.com.

${ }^{2}$ Acadêmica de Medicina. Universidade Estadual de Ponta Grossa. E-mail: dornellescf@gmail.com.

3 Farmacêutico. Doutor em Saúde Coletiva. Universidade Estadual de Ponta Grossa. E-mail: erildomuller@hotmail.com.

${ }^{4}$ Acadêmico de Medicina. Universidade Estadual de Ponta Grossa. E-mail: torrani.allan@gmail.com.

${ }^{5}$ Acadêmico de Medicina. Universidade Estadual de Ponta Grossa. E-mail: alisson_pupulim@hotmail.com.

${ }^{6}$ Acadêmico de Medicina. Universidade Estadual de Ponta Grossa. E-mail: carlosrorypucci@hotmail.com.

${ }^{7}$ Acadêmica de Medicina. Universidade Estadual de Ponta Grossa. E-mail: maki_naka_7@hotmail.com.

${ }^{8}$ Acadêmico de Medicina. Universidade Estadual de Ponta Grossa. E-mail: matheougusto@hotmail.com.
} 


\section{ASSOCIATED FACTORS}

ABSTRACT: Smoking is related to several diseases and its treatment brings benefits to active and passive smokers. This study aimed to analyze the treatment results in smokers and possible associated factors. A retrospective study was carried out, with data from 62 patients treated in a university extension project. Socioeconomic, clinical and smoking data were collected. Data on smoking cessation outcomes were also collected during the treatment period and after treatment (mean time $8.0 \pm 2,0$ months after initiation of treatment). Statistical analyzes were performed using the Chi-square test and Fisher Exact test. The level of significance was 5\%. During the treatment period $51.6 \%$ of the patients stopped smoking and $37.1 \%$ reduced the number of cigarettes per day. Out of the 54 patients who responded to telephone contacts after treatment, $31.5 \%$ remained smoking-free, $27.8 \%$ were smoking less cigarettes per day and $16.6 \%$ relapsed. At least 5 times during treatment $(p<0,001)$ and high or very high nicotine dependence level $(p=0,03)$ were associated with non-stop smoking during treatment. A good proportion of smoking cessation was observed during and after treatment. An encouragement must be given for patients to attend treatment meetings, with special attention for those with high or very high nicotine dependence.

Keywords: Tobacco use disorder. Tobacco use cessation. Tobacco use cessation products. National Program of Tobacco Control.

\section{INTRODUÇÃO}

De acordo com a Organização Mundial de Saúde, 21\% da população adulta era tabagista no ano de 2013, 950 milhões de homens e 177 milhões de mulheres, sendo que em países emergentes, como o Brasil, essa prevalência chegava a $25 \%$ dos adultos (World Health Organization, 2015). O tabagismo é considerado pela Associação Americana de Psiquiatria um transtorno que causa comprometimento clínico e pode levar os pacientes ao fracasso de obrigações familiares ou profissionais além de poder causar problemas sociais (American Psychiatric Association, 2014).

O uso do tabaco é o maior fator de risco evitável para as doenças crônicas não transmissíveis, principalmente cânceres, diabetes, doenças cardiovasculares e doenças crônicas pulmonares (World Health Organization, 2015). É responsável pela morte de cerca de cinco milhões de pessoas por ano em todo o mundo, representando mais de $20 \%$ de todas as mortes de homens adultos e $5 \%$ das mortes de mulheres adultas. No século 20 ocorreram 100 milhões de mortes por tabaco. No século 21, o tabaco deverá matar cerca de um bilhão de pessoas, principalmente em países de baixa e média renda (Jha et al., 2015). Além disso, os efeitos prejudiciais do tabagismo não se restringem apenas aos fumantes, podendo também afetar os fumantes passivos (Reichert. et al., 2008).

Por outro lado, os maiores benefícios individuais da cessação do tabagismo se traduzem em grandes reduções nas mortes por todas as causas atribuíveis ao tabagismo e do câncer na população em geral (Jha et al., 2015). Também melhora a qualidade de vida e a autoestima do fumante após a cessação (Reichert. et al., 2008).

Nos últimos anos tem havido redução da prevalência de tabagistas no Brasil, devido 
a uma combinação de políticas de controle do tabagismo, como as restrições publicitárias, a proibição do uso do tabaco em lugares públicos e aumento dos impostos (GBD 2015 Tobacco Collaborators, 2017). Contudo, existem poucos estudos nacionais que avaliaram os resultados do tratamento combinado para cessação do tabagismo, sendo que os trabalhos publicados apresentam resultados de sucesso terapêutico variando de $31,0 \%$ até 82,9\% (Rodrigues et al., 2016; França et al., 2015; Goyatá et al., 2014; Azevedo et al., 2009; Sales et al., 2006; Rodrigues et al., 2015).

Além disso, os fatores associados aos resultados do tratamento do tabagismo descritos na literatura são diversos, como a escolaridade (Peña et al., 2016; Peixoto et al; 2007), idade, estado civil (Peixoto et al., 2007), grau de dependência à nicotina (França et al., 2015; Rodrigues et al., 2015; Peña, et al., 2016), frequência aos encontros de terapia(Rodrigues et al., 2016; Goyatá et al., 2014), terapia de manutenção e gatilhos de recaída (França et al., 2015), sintomas psiquiátricas e síndrome de abstinência (Azevedo et al., 2009), parceiro fumante (Walsh et al., 2007), uso de medicação (Sales et al., 2006), número de hospitalizações e de consultas no último ano e número de condições crônicas (Peixoto et al., 2007).

Os objetivos desse estudo foram analisar os resultados do tratamento e identificar possíveis fatores associados a não cessação do tabagismo em pacientes atendidos em um projeto de extensão universitária.

\section{MATERIAL E MÉTODOS}

Estudo retrospectivo e com abordagem quantitativa. Foram analisados os dados de 62 prontuários de pacientes tabagistas tratados de março a dezembro de 2016 em um projeto de extensão em uma universidade no Sul do Brasil.

O projeto de extensão é composto por uma equipe interdisciplinar treinada, formada por docentes dos cursos de enfermagem, farmácia e medicina e acadêmicos de graduação desses cursos. Está vinculado ao projeto intitulado "Programa Nacional de Controle do Tabagismo", para abordagem e tratamento do tabagismo no Sistema Único de Saúde, desenvolvido pelo Instituto Nacional do Câncer (INCA) e Ministério da Saúde. Tem como objetivos principais estimular o abandono do fumo entre os dependentes do tabaco e promover educação em saúde sobre o tabagismo.

O programa prevê quatro encontros iniciais, uma vez por semana, com duração média de 2 horas. Como parte da abordagem cognitivo-comportamental, em cada um dos quatro encontros iniciais o participante recebe um livreto elaborado pelo Ministério da Saúde, chamado "Deixando de fumar sem mistérios", que traz orientações sobre todo o processo para deixar o tabagismo (Brasil, 2004). O primeiro livreto é intitulado "Entender por que se fuma e como isso afeta a saúde", o segundo "Os primeiros dias sem fumar", o terceiro "Como vencer os obstáculos para permanecer sem fumar" e o quarto "Benefícios obtidos após parar de fumar". 
No primeiro encontro também são realizadas as apresentações dos pacientes e da equipe de saúde, são preenchidas as fichas dos pacientes com informações sociodemográficas, histórico de doenças, uso de medicamentos e o histórico tabágico. Também são preenchidos questionários para avaliar o grau de dependência à nicotina e grau de motivação para parar de fumar.

A abordagem cognitivo-comportamental, utilizada no projeto de extensão enfatiza a motivação e as mudanças comportamentais indispensáveis para a cessação do tabagismo, além dos objetivos da cessação, benefícios, gatilhos, frustrações, mecanismos automáticos, mecanismos de gratificação, ambivalência e o desenvolvimento de um plano de enfrentamento sobre as causas mais comuns de recaída. A terapia medicamentosa visa reduzir os sintomas da síndrome de abstinência (Silva et al., 2016).

A indicação para o tratamento medicamentoso considera os hábitos tabágicos e a evolução do paciente durante o tratamento, sendo indicado ou não o uso de drogas como a reposição de nicotina e/ou antidepressivo. Os medicamentos considerados como primeira linha no tratamento da dependência à nicotina e utilizados no Brasil são a terapia de reposição de nicotina, através do adesivo transdérmico, goma de mascar e pastilha, e o cloridrato de bupropiona (Brasil, 2016).

Após as quatro primeiras semanas, os pacientes participam de encontros quinzenais por três meses, seguidos de encontros de acompanhamento. Todo o tratamento oferecido ao paciente é gratuito.

Foram coletados dos prontuários dos pacientes os dados sociodemográficos de idade, gênero, estado civil, anos de estudo e trabalho remunerado. A variável clínicas foi o uso de medicamento psicotrópico. Referente ao tabagismo, foram coletados dados sobre o tempo do tabagismo, idade de início do tabagismo, se residia com outra(s) pessoa(s) tabagista(s), grau de dependência à nicotina (Teste de Fagerström), tipo de tratamento(s) realizado(s) e frequência de presenças durante o tratamento.

O grau de dependência à nicotina foi avaliado pelo Teste de Fagerström (Heatherton et al., 1991). O teste consta de seis questões e para cada alternativa de resposta existe uma pontuação. A soma dos pontos de cada alternativa escolhida permitirá a avaliação do grau de dependência à nicotina. Assim, o grau de dependência é avaliado segundo as seguintes pontuações: 0-2 pontos=muito baixo; 3-4 pontos= baixo; 5 pontos=médio; 6-7 pontos=elevado; 8-10 pontos=muito elevado. Com um resultado acima de 6 pontos, provavelmente o paciente terá síndrome de abstinência ao deixar de fumar.

Também foram coletadas as informações sobre o estado dos pacientes com relação ao tabagismo após o tratamento. Essas informações referem-se às respostas dadas pelos pacientes por meio de ligações telefônicas realizadas durante o mês de fevereiro de 2017, como parte das atividades do projeto de extensão, registradas em prontuário. As informações incluíam se o paciente havia permanecido sem fumar, se havia voltado a fumar ou se continuava fumando, bem como se havia reduzido o número de cigarros utilizados. 
Foram utilizadas medidas estatísticas descritivas para apresentar as características dos participantes, os motivos dos pacientes para desejar parar de fumar e os resultados durante e após o tratamento. O teste Qui-Quadrado de Pearson e o teste Exato de Fisher foram usados para avaliar a associação das diversas variáveis com a variável de desfecho (pacientes que pararam e não pararam de fumar). O nível de significância adotado foi de $5 \%$.

Os dados foram processados em planilha eletrônica no Programa Microsoft Office Excel${ }^{\circledR} 2010$ para Windows ${ }^{\circledR}$. A análise estatística foi obtida com auxílio do programa Statistical Package for Social Science (SPSS), versão 15.0. O nível de significância utilizado foi de $5 \%$.

O projeto foi aprovado pela Comissão de Ética em Pesquisa da instituição de ensino sob o Parecer número 1.055.794, CAAE: 43523515.8.0000.0105.

\section{RESULTADOS}

Dentre os 62 pacientes que realizaram tratamento em 2016, 50\% eram do gênero feminino, a média de idade no início do tratamento foi de $45,9 \pm 12,0$ anos e do início do tabagismo foi de $18,7 \pm 8,8$. A média do tempo de tabagismo foi de $27,2 \pm 13,2$ anos. 0 número de cigarros consumidos ao dia em média foi de $22,0 \pm 12,4$ e da carga tabágica (anos-maço) de $32,1 \pm 28,7$.

Durante o tratamento, 32 pacientes $(51,6 \%)$ cessaram o tabagismo, $23(37,1 \%)$ reduziram o número de cigarros consumidos por dia e apenas $7(11,3 \%)$ não pararam de fumar. Após o tratamento, foi possível contato com 54 pacientes, com tempo médio de seguimento a partir do início do tratamento de 8,0 meses $\pm 2,0$. Desses 54 pacientes, 17 $(31,5 \%)$ permaneciam sem fumar, $15(27,8 \%)$ não pararam de fumar mas reduziram o número de cigarros ao dia, $13(24,1 \%)$ não pararam e permaneceram fumando e 9 (16,5\%) sofreram recaída (Tabela 1).

Tabela 1 - Distribuição dos pacientes durante e após o tratamento de acordo com o resultado do tratamento antitabagismo em um projeto de extensão universitária, 2016 ( $\mathrm{N}=62$ e 54, respectivamente)

\begin{tabular}{|c|c|c|c|}
\hline \multicolumn{2}{|l|}{ Durante o tratamento $(\mathrm{N}=62)$} & \multicolumn{2}{|l|}{ Após o tratamento* $(\mathrm{N}=54)^{\star *}$} \\
\hline Resultado & $\mathrm{N}(\%)$ & Resultado & $N(\%)$ \\
\hline Parou de fumar & $32(51,6)$ & Parou e continua sem fumar & $17(31,5)$ \\
\hline $\begin{array}{l}\text { Não parou, mas reduziu o número de } \\
\text { cigarros }\end{array}$ & $23(37,1)$ & $\begin{array}{l}\text { Não parou, mas reduziu o número de } \\
\text { cigarros }\end{array}$ & $15(27,8)$ \\
\hline $\begin{array}{l}\text { Não parou e manteve o número de } \\
\text { cigarros }\end{array}$ & $7(11,3)$ & $\begin{array}{l}\text { Não parou e manteve o número de } \\
\text { cigarros }\end{array}$ & $13(24,1)$ \\
\hline- & - & Recaída & $9(16,6)$ \\
\hline
\end{tabular}

* Tempo médio da avaliação de 8,0 $\pm 2,0$ meses após o início do tratamento

** Oito pacientes não responderam aos contatos telefônicos

Fonte: Os autores (2017)

Os dados socioeconômicos e clínicos para o total de pacientes e a comparação 
entre os pacientes que pararam e não pararam de fumar são apresentados na Tabela 2. Com relação ao total de pacientes, não houve diferença em relação ao número de homens e mulheres e o estado civil. A maioria tinha até 50 anos de idade no início do tratamento, até 8 anos de estudo e com trabalho remunerado ou aposentada. Um total de $28,5 \%$ dos pacientes utilizavam medicamento psicotrópico.

A Tabela 2 também apresenta a comparação entre os pacientes que pararam e não pararam de fumar, sendo que não foi observada diferença significativa para idade no início do tratamento, gênero, estado civil, anos de estudo, trabalho remunerado e uso de medicamento psicotrópico.

Tabela 2 - Distribuição dos pacientes segundo variáveis socioeconômicas e clínicas para o total de pacientes e a comparação entre os pacientes que não pararam e pararam de fumar durante o tratamento, $2016(\mathrm{~N}=62)$.

\begin{tabular}{|c|c|c|c|c|}
\hline Variáveis & $\begin{array}{l}\text { Total } \\
(\mathrm{N}=62) \\
\mathrm{N}(\%) \\
\end{array}$ & $\begin{array}{l}\text { Não parou de } \\
\text { fumar } \\
(\mathrm{N}=30) \\
\mathrm{N}(\%) \\
\end{array}$ & $\begin{array}{l}\text { Parou de } \\
\text { fumar } \\
(\mathrm{N}=32) \\
\mathrm{N}(\%) \\
\end{array}$ & $p$ \\
\hline \multicolumn{5}{|l|}{ Idade no início do tratamento } \\
\hline Até 50 anos & $37(59,7)$ & $15(50,0)$ & $22(68,8)$ & \multirow{2}{*}{$0,13^{*}$} \\
\hline Mais de 50 & $25(40,3)$ & $15(50,0)$ & $10(31,2)$ & \\
\hline \multicolumn{5}{|l|}{ Gênero } \\
\hline Feminino & $31(50)$ & $18(60,0)$ & $13(40,6)$ & \multirow[t]{2}{*}{$0,13^{*}$} \\
\hline Masculino & $31(50)$ & $12(40,0)$ & $19(59,4)$ & \\
\hline \multicolumn{5}{|l|}{ Estado Civil } \\
\hline Sem companheiro(a) & $31(50)$ & $17(56,7)$ & $14(43,8)$ & \multirow{2}{*}{$0,31^{*}$} \\
\hline Com companheiro(a) & $31(50)$ & $13(43,3)$ & $18(56,2)$ & \\
\hline \multicolumn{5}{|l|}{ Anos de estudo\# } \\
\hline Até 8 & $31(50,8)$ & $17(56,7)$ & $14(43,8)$ & \multirow[t]{2}{*}{$0,37^{*}$} \\
\hline Mais de 8 & $30(49,2)$ & $13(43,3)$ & $17(53,2)$ & \\
\hline \multicolumn{5}{|l|}{ Trabalho remunerado } \\
\hline Sem trabalho remunerado & $12(19,4)$ & $7(23,3)$ & $5(15,6)$ & \multirow[t]{2}{*}{$0,44^{*}$} \\
\hline Trabalho remunerado ou aposentado & $50(80,6)$ & $23(76,7)$ & $27(8,4)$ & \\
\hline \multicolumn{5}{|l|}{ Uso de medicamento psicotrópico } \\
\hline Sim & $16(25,8)$ & $10(33,3)$ & $6(18,8)$ & \multirow[t]{2}{*}{$0,19^{* *}$} \\
\hline Não & $46(74,2)$ & $20(66,7)$ & $26(81,2)$ & \\
\hline
\end{tabular}

Quanto às variáveis relacionadas ao tabagismo, a maioria dos pacientes tinha mais de 20 anos de tempo de tabagismo, iniciou o tabagismo antes dos 18 anos, residia com tabagista(s) e apresentava grau de dependência à nicotina elevado ou muito elevado. Com relação ao tratamento, a grande maioria dos pacientes $(90,3 \%)$ recebeu tratamento medicamentoso para cessação do tabagismo, predominando a reposição de nicotina $(85,47)$. Pouco mais da metade dos pacientes teve uma frequência de presenças aos encontros durante o tratamento de 5 vezes ou mais (Tabela 3). Todos os pacientes participaram das atividades referentes à abordagem cognitivo-comportamental.

Comparando-se os pacientes que pararam e não pararam de fumar, o tempo de tabagismo, a idade no início do tabagismo, residir com tabagista(s), a realização de tratamento medicamentoso, o tipo de tratamento realizado e tipo da reposição de nicotina 
não apresentaram diferenças significativas. As variáveis grau de dependência à nicotina elevado ou muito elevado $(p=0,03)$ e frequência de presenças aos encontros durante o tratamento inferior a 5 vezes $(p<0,001)$ foram associadas à não parar de fumar (Tabela 3$)$.

Tabela 3 - Distribuição dos pacientes segundo variáveis do tabagismo para o total de pacientes e a comparação entre os pacientes que não pararam e pararam de fumar durante o tratamento, 2016 (N=62).

\begin{tabular}{|c|c|c|c|c|}
\hline \multirow[t]{2}{*}{ Variáveis } & $\begin{array}{l}\text { Total } \\
(\mathrm{N}=62)\end{array}$ & $\begin{array}{l}\text { Não parou } \\
\text { de fumar } \\
(\mathrm{N}=30)\end{array}$ & $\begin{array}{l}\text { Parou de } \\
\text { fumar } \\
(\mathrm{N}=32)\end{array}$ & \multirow[t]{2}{*}{$p$} \\
\hline & $\mathbf{N}(\%)$ & $\mathbf{N}(\%)$ & N (\%) & \\
\hline \multicolumn{5}{|l|}{ Tempo de tabagismo em anos } \\
\hline Até 20 & $23(37,1)$ & $8(26,7)$ & $15(46,9)$ & \multirow[t]{2}{*}{$0,10^{*}$} \\
\hline Mais de 20 & $39(62,9)$ & $22(73,3)$ & $17(53,1)$ & \\
\hline \multicolumn{5}{|c|}{ Idade de início do tabagismo em anos } \\
\hline Antes dos 18 & $37(59,7)$ & $20(66,7)$ & $17(53,1)$ & \multirow[t]{2}{*}{$0,28^{*}$} \\
\hline 18 ou mais & $25(40,3)$ & $10(33,3)$ & $15(46,9)$ & \\
\hline \multicolumn{5}{|l|}{ Reside com tabagista(s) } \\
\hline Sim & $34(54,8)$ & $20(66,7)$ & $14(43,8)$ & \multirow[t]{2}{*}{$0,07^{*}$} \\
\hline Não & $28(45,2)$ & $10(33,3)$ & $18(56,2)$ & \\
\hline \multicolumn{5}{|c|}{ Grau de dependência (Teste de Fargeström) } \\
\hline Elevado ou muito elevado & $39(62,9)$ & $23(76,7)$ & $16(50,0)$ & \multirow[t]{2}{*}{$0,03^{*}$} \\
\hline Muito baixo, baixo ou médio & $23(37,1)$ & $7(23,3)$ & $16(50,0)$ & \\
\hline \multicolumn{5}{|l|}{ Tratamento medicamentoso } \\
\hline Sim & $56(90,3)$ & $25(83,3)$ & $31(96,9)$ & \multirow[t]{2}{*}{$0,10^{* *}$} \\
\hline Não & $6(9,7)$ & $5(16,7)$ & $1(3,1)$ & \\
\hline \multicolumn{5}{|l|}{ Tipo de tratamento } \\
\hline Bupropiona & $8(14,3)$ & $2(6,7)$ & $6(18,8)$ & \multirow[t]{2}{*}{$0,23^{*}$} \\
\hline Reposição de nicotina & $48(85,7)$ & $23(76,7)$ & $25(78,2)$ & \\
\hline \multicolumn{5}{|l|}{ Reposição de nicotina } \\
\hline Adesivo+goma & $29(60,4)$ & $14(46,7)$ & $15(46,9)$ & \multirow[t]{2}{*}{$0,95^{*}$} \\
\hline Adesivo & $19(39,6)$ & $9(30,0)$ & $10(31,3)$ & \\
\hline \multicolumn{5}{|c|}{$\begin{array}{l}\text { Frequência de presenças aos encontros durante o } \\
\text { tratamento }\end{array}$} \\
\hline Menos de 5 vezes & $27(43,5)$ & $19(63,3)$ & $8(25,0)$ & \multirow[t]{2}{*}{$<0,001$} \\
\hline 5 vezes ou mais & $35(56,5)$ & $11(36,7)$ & $24(75,0)$ & \\
\hline
\end{tabular}

*Teste Qui-quadrado ${ }^{* *}$ Teste Exato de Fisher

Fonte: os autores (2017)

\section{DISCUSSÃO}

Dentre os 62 pacientes tabagistas tratados em 2016, 51,6\% pararam de fumar durante o tratamento, considerado um bom resultado quando comparado aos resultados publicados de outras pesquisas (Rodrigues et al., 2016; Sales et al., 2006; Rodrigues et al., 2015). Contudo, percentuais de sucesso terapêutico maiores foram relatados (França et al., 2015; Goyatá et al., 2014; Azevedo et al., 2009; Peña et al., 2016).

Além dos pacientes que pararam de fumar, $37,1 \%$ reduziram o número de cigarros fumados por dia. Quando foi realizada a avaliação posterior ao tratamento em 54 pacientes, com média de acompanhamento de $8 \pm 2,0$ meses, observou-se que 17 pacientes $(31,5 \%)$ permaneciam sem fumar e $15(27,8 \%)$ estavam fumando número menor de cigarros que no início do tratamento. 
Os pacientes participantes do projeto receberam tratamento combinado, que inclui a abordagem cognitivo-comportamental e tratamento farmacológico. Uma recente revisão analisou ensaios controlados randomizados ou quase randomizados em que todos os participantes receberam farmacoterapia para cessação do tabagismo e as condições diferiram pela quantidade de suporte comportamental. Os autores concluíram que fornecer suporte comportamental pessoalmente ou por telefone para pessoas que usam farmacoterapia para parar de fumar tem um efeito pequeno, mas importante. $O$ aumento na quantidade de suporte comportamental provavelmente aumentará a chance de sucesso em cerca de $10 \%$ a 25\%, com base em uma estimativa agregada de 47 ensaios clínicos analisados (Stead et al., 2015).

Outra revisão foi realizada com base em 41 estudos, entre ensaios controlados randomizados ou quase randomizados, com um total de mais de 20.000 participantes. A maioria dos estudos forneceu terapia de reposição de nicotina e o suporte comportamental foi oferecido por especialistas em aconselhamento para cessação, entre quatro e oito sessões. Os autores concluíram que intervenções que combinam farmacoterapia e suporte comportamental aumentam o sucesso de cessação do tabagismo em comparação com intervenção mínima ou cuidados habituais (Stead; Lancaster, 2012).

Os percentuais de cessação do tabagismo apresentam grandes variações na literatura (Rodrigues et al., 2016; França et al., 2015; Goyatá et al., 2014; Azevedo et al., 2009; Sales et al., 2006; Rodrigues et al., 2015; Peña et al., 2016).

Por exemplo, percentual de sucesso durante o ano de tratamento bem superior ao encontrado neste estudo, de $82,9 \%$, foi relatado em estudo com 35 pacientes tratados em um ambulatório municipal no Sul de Minas Gerais, submetidos a tratamento combinado e realizado por uma por equipe multidisciplinar (Goyatá et al., 2014). Taxa de sucesso também maior a deste estudo, de $79,1 \%$ durante o tratamento combinado e de $62 \%$ após 25 meses, foi relatada em análise de 171 pacientes, sendo $47 \%$ deles com sintomas atuais de ansiedade ou depressão e 28,6\% com antecedentes de uso de drogas de abuso ou de etilismo. Esses pacientes participaram de um grupo motivacional (nem todos puderam participar) em um ambulatório para tratamento de dependência a substâncias psicoativas multidisciplinar, na cidade de Campinas (Azevedo et al., 2009).

Outro exemplo de percentual maior de cessação do tabagismo foi relatado em um estudo realizado na cidade de Belém, onde $75 \%$ dos 532 pacientes pararam de fumar. 0 tratamento consistiu na abordagem cognitivo-comportamental isolada ou em conjunto com o tratamento medicamentoso. Os autores atribuíram a alta taxa de sucesso ao fato dos pacientes serem tabagistas de longa data e à alta carga tabágica, com médias de 32,4 anos e 30 maços/ano, respectivamente, o que teria feito com que os pacientes se engajassem mais efetivamente no tratamento (França et al., 2015).

Dois relatos apresentaram percentuais de sucesso no tratamento semelhantes aos deste estudo. Um deles, realizado no Ceará, utilizou a associação de tratamento medicamentoso e a abordagem cognitivo-comportamental para o tratamento de tabagistas, conforme as diretrizes preconizadas pelo INCA, sendo que dos 258 pacientes analisados, 
que se encontravam há pelo menos um ano no programa, a taxa de sucesso terapêutico foi de 50,8\% (Sales et al., 2006). Outro analisou as características dos tabagistas que buscaram tratamento em uma unidade básica de saúde de uma comunidade carente na cidade do Rio de Janeiro. O tratamento consistiu em terapia cognitivo-comportamental e medicamentosa. A maioria das pacientes eram mulheres $(71 \%)$ e cerca de metade dos participantes deixaram de fumar ( $n=125,51 \%)$ (Rodrigues et al., 2015).

Pesquisadores chilenos avaliaram retrospectivamente 281 pacientes submetidos ao tratamento combinado realizado por equipe interdisciplinar, utilizando apenas a vareniclina como tratamento medicamentoso. A taxa de sucesso após um ano do tratamento foi de 53,4\%. O contato telefônico após o final do tratamento realizado pelo mesmo profissional, foi um fator que auxiliou no resultado, em função de melhor vínculo entre profissional-paciente (Peña et al., 2016).

Em recente estudo realizado no Rio de Janeiro com 84 pacientes, utilizando abordagem cognitivo-comportamental e terapia de reposição de nicotina, 40,5\% dos participantes conseguiram parar de fumar por pelo menos três meses, percentual menor do que o observado neste estudo. Apenas 31\% dos pacientes que iniciaram o tratamento obtiveram manutenção da cessação após um ano do final do tratamento e $19 \%$ se mantiveram após 2 anos. Os autores consideraram que o sucesso a longo prazo poderia ter sido maior se os pacientes pudessem refazer o tratamento, tendo em vista que as vagas eram limitadas e se dava preferência para aqueles que nunca haviam participado (Rodrigues et al., 2016).

Apesar dos estudos citados terem utilizado o tratamento combinado de terapia e medicamentos para cessação do tabagismo, foram observadas muitas variações com relação aos resultados dos tratamentos, especialmente sobre o percentual de cessação tabágica. Diferenças relacionadas às características da amostra, do acesso ao serviço, dos tipos de tratamentos utilizados, de como a abordagem da terapia comportamental foi conduzida e do momento em que a avaliação foi realizada podem ter influenciado os resultados, bem como diferenças regionais ou limitações metodológicas relativas aos delineamentos utilizados.

Neste estudo, em um tempo médio de seguimento de 8,0 meses após o início do tratamento, a recaída ocorreu em 9 pacientes $(16,6 \%)$, dentre os 54 pacientes contatados. Na avaliação realizada com pacientes ambulatoriais em um município de Minas Gerais, apesar do sucesso com terapia combinada de $82,9 \%$ durante o ano de tratamento, na avaliação após 18 meses a recaída foi de 41,3\%(Goyatá et al., 2014). Taxa de recaída menor, de $17,8 \%$, porém observada para qualquer fase do tratamento, foi descrita no estudo já citado realizado em Fortaleza, no Ceará. Também se observou uma associação significativamente maior de recaída nos pacientes que necessitaram de terapia complementar, como a acupuntura e/ou atendimento psicológico individual, sugerindo que aqueles com maior dependência psicológica podem apresentar recaídas com maior frequência (Sales et al., 2006). Ainda uma taxa de recaída de 10,0\%, foi encontrada no estudo realizado em Campinas, que pode ter ocorrido pelo fato dos pacientes não serem 
submetidos a avaliações posteriores, facilitando assim o retorno dos hábitos tabágicos. Além disso, os sintomas psiquiátricos e menor número de sessões no grupo de motivação foram relacionados à recaída (Azevedo et al., 2009).

$\mathrm{Na}$ comparação entre os pacientes que pararam e não pararam de fumar analisados neste estudo, a frequência de presença aos encontros durante o tratamento inferior a 5 vezes foi associada a não parar de fumar. De modo semelhante, a frequência aos encontros foi uma variável com associação ao desfecho positivo do tratamento, com aumento de $34 \%, 48 \%$ e $97 \%$ na chance dos pacientes pararem de fumar por pelo menos seis, 12 e 24 meses, respectivamente, a cada nova sessão que o paciente participou. Aqueles pacientes que compareceram a três ou mais encontros tiveram risco $79 \%$ menor de voltar a fumar do que aqueles que foram a menos de três encontros (Rodrigues et al., 2016).

No estudo realizado em um ambulatório no sul de Minas Gerais, os resultados demonstraram como fator de sucesso na cessação do tabagismo a participação em quatro a seis sessões do programa. Os autores comentam parecer ser muito importante 0 desenvolvimento de ações sistematizadas para que se mantenha fluxo contínuo das informações e sensibilização dos pacientes (Goyatá et al., 2014). Acredita-se que uma taxa de sucesso de $62 \%$ após 25 meses do tratamento, considerada alta, deveu-se ao comparecimento mensal dos pacientes a um grupo de tratamento, até completar um ano da cessação do tabagismo e às sessões motivacionais. Os autores sugerem a inclusão e ampliação de estratégias motivacionais no processo anterior à tentativa de cessação, especialmente em serviços públicos onde pode haver limitações na disponibilidade de fármacos (Azevedo et al., 2009).

O grau de dependência à nicotina avaliada pelo Teste de Fagerström como elevado ou muito elevado foi outra variável associada a não parar de fumar neste estudo. A associação entre insucesso do tratamento e alta dependência à nicotina pelo escore de Fagëstrom também foi descrita na literatura (Peña et al., 2016). Além disso, dependência química mais baixa, terapia de manutenção e ausência de gatilhos de recaída foram significativamente associados à cessação do tabagismo (França et al., 2015).

O Teste de Fagerström é muito utilizado para avaliação da dependência à nicotina (Reichert et al., 2008), sendo que quanto maior o escore, maior será o grau de dependência. Fumantes com escore maior ou igual a 7 são considerados os mais dependentes e provavelmente terão menor motivação para parar de fumar pela menor confiança na habilidade de superar os sintomas da abstinência (Araújo et al., 2004).

A síndrome de abstinência é considerada uma das principais causas da recaída. Portanto, o tratamento e o seguimento adequados desses fumantes são fundamentais para bons resultados nos programas de cessação (Reichert et al., 2008).

Os esforços concentrados para implementar diversas políticas e programas de controle de tabaco possivelmente contribuíram para a redução na prevalência de tabagismo no Brasil nas últimas décadas (GBD 2015 Tobacco Collaborators, 2017; Silva et al., 2014) 
Apesar disso, o tabagismo continua sendo um grave problema de saúde pública e exige ações políticas e controle social para a sua desnormatização (Silva et al., 2014. Possivelmente muitas décadas serão necessárias para que essa diminuição da prevalência do tabagismo modifique o cenário epidemiológico atual no país.

Importante ressaltar que o tratamento para cessação do tabagismo tem um bom desempenho com relação ao custo para cada paciente que deixa de fumar. Dessa forma, considerando a magnitude da carga tabágica, o tratamento para cessação do tabagismo no Brasil deve ser considerado uma prioridade quando da alocação de recursos para saúde (Mendes et al., 2017).

Este estudo apresentou limitações, como o caráter transversal que não permite estabelecer relações de causalidade e a utilização de prontuários como fonte de dados, com a possibilidade de sub-registros. Além disso, as informações sobre a evolução dos pacientes quanto ao consumo de cigarros era obtida por meio de autorrelato. Apesar das limitações, esse estudo apresentou resultados de pacientes durante e após o tratamento realizado por equipe interdisciplinar, com a identificação de fatores associados a não parar de fumar durante o tratamento, que podem contribuir para o melhor entendimento sobre os aspectos que envolvem os resultados do tratamento do tabagismo.

\section{CONSIDERAÇÕES FINAIS}

Observou-se neste estudo uma boa proporção de cessação do tabagismo entre os pacientes durante e após o tratamento. Incentivo deve ser dado para que os pacientes compareçam aos encontros de tratamento, com atenção especial para aqueles com grau de dependência elevado ou muito elevado.

Os fatores determinantes para o consumo de cigarros e para a não cessação do tabagismo sejam eles comportamentais, econômicos ou sociais, devem ser estudados tanto em curto como em longo prazos, pois podem gerar informações úteis para orientar políticas de saúde. Essas informações devem ser parte de um sistema de vigilância de dados, orientando a implantação de estratégias de enfrentamento adequadas, tanto nos sistemas de saúde pública como suplementar.

\section{REFERÊNCIAS}

American Psychiatric Association. Manual diagnóstico e estatístico de transtornos mentais: DSM-5. 5ํㅡㄹ ed. Porto Alegre: Artmed, 2014.

Araújo, A. J. et al. Diretrizes para Cessação do Tabagismo. Jornal Brasileiro de Pneumologia, v. 30, n. 2, S1-S76, 2004.

Azevedo, R. C. S. et al. Grupo terapêutico para tabagistas: resultados após seguimento de dois anos. Revista da Associação Médica Brasileira, v.55, n. 5, p. 593-596, 2009. 
Brasil. Ministério da Saúde. Instituto Nacional de Câncer - INCA. Coordenação de Prevenção e Vigilância (CONPREV). Deixando de Fumar sem Mistérios. Rio de Janeiro: MS/INCA, 2004. Disponível em: <http://www.ensp.fiocruz.br/portalensp/informe/site/materia/detalhe/12633>. Acesso em 08 nov. 2017.

Brasil. Ministério da Saúde. Portaria nำ761, de 21 de junho de 2016. Valida as orientações técnicas do tratamento do tabagismo constantes no Protocolo Clínico e Diretrizes Terapêuticas - Dependência à Nicotina. Diário Oficial [da] República Federativa do Brasil. 2016, v. 15, n. 1, p. 68.

França, S. A. S. et al. Factors associated with smoking cessation. Revista de Saúde Pública, v. 40, n. 10, 2015. Disponível em:

<http://www.scielo.br/scielo.php?script=sci_arttext\&pid=S0034$89102015000100203 \&$ Ing=em>. Acesso em 04 ago. 2017.

GBD 2015 Tobacco Collaborators. Smoking prevalence and attributable disease burden in 195 countries and territories, 1990-2015: a systematic analysis from the Global Burden of Disease Study 2015. Lancet, v. 389, n. 10082, p. 1885-1906, 2017.

Goyatá, S. L. T. et al. Impacto do programa de apoio ao tabagista de um município do Sul de Minas Gerais, Brasil. Ciencia y Enfermería, v. 20, n, 1, p. 77-88, 2014.

Heatherton, T. F. et al. The Fagerström Test for Nicotine Dependence: a revision of the Fagerström Tolerance Questionnaire. British Journal of Addiction, v. 86, n. 9, p. 11191127, 1991.

Jha, P. et al. Global Hazards of Tobacco and the Benefits of Smoking Cessation and Tobacco Taxes. Source Cancer: Disease Control Priorities, Third Edition (Volume 3). Washington (DC): The International Bank for Reconstruction and Development / The World Bank, 2015, Cap. 10.

Mendes, A. C. R. et al. Costs of the Smoking Cessation Program in Brazil. Revista de Saúde Pública, v. 50, n. 66, 2016. Disponível em: < https://www.ncbi.nlm.nih.gov/pmc/articles/PMC5117528/>. Acesso em 04 ago. 2017.

Peixoto, S. V. et al. Fatores associados ao índice de cessação do hábito de fumar em duas diferentes populações adultas (Projetos Bambuí e Belo Horizonte). Cadernos de Saúde Pública, v. 23, n. 6, p. 1319-1328, 2007.

Peña, P. et al. Factores asociados con el éxito de la terapia antitabaco en pacientes tratados con vareniclina: 10 años de experiencia en un programa multidisciplinario. Revista Médica do Chile, v. 144, p. 965-971, 2016.

Reichert, J. et al. Diretrizes para cessação do tabagismo. Jornal Brasileiro de Pneumologia, v. 34, n. 10, p. 845-880, 2008.

Rodrigues, N. C. et al. Profile of Brazilian smokers in the National Program for Tobacco Control. Revista Brasileira de Psiquiatria, v. 37, n.2, p. 150-154, 2015.

Rodrigues, et al. Long-term e ects of smoking cessation support in primary care: results of a two-year longitudinal study in Brazil. Jornal Brasileiro de Psiquiatria, v. 65, n. 2, p. 174178, 2016. 
Sales, M. P. U. et al. Ambulatório de apoio ao tabagista no Ceará: perfil dos pacientes e fatores associados ao sucesso terapêutico. Jornal Brasileiro de Pneumologia, v. 32, p. 4147, 2006.

Silva, S. T. et al. Combate ao Tabagismo no Brasil: a importância estratégica das ações governamentais. Ciência \& Saúde Coletiva, v. 19, n. 2, p. 539-552, 2014.

Silva, L. C. C. et al. Comissão de Tabagismo da SBPT. Controle do tabagismo: desafios e conquistas. Jornal Brasileiro de Pneumologia, v. 42, n. 4, p. 290-298, 2016.

Stead, L. F.; Lancaster, T. Combined pharmacotherapy and behavioural interventions for smoking cessation. Cochrane Database Systematic Review, 2012, v. 10, CD008286.

Stead, L. F. et al. Additional behavioural support as an adjunct to pharmacotherapy for smoking cessation. Cochrane Database Systmatic Review, 2015, n. 12, v. 10, CD009670.

Walsh, P. M. et al. Effects of partner smoking status and gender on long term abstinence rates of patients receiving smoking cessation treatment. Addictive Behaviors, v. 32, n. 1, p. 128-136, 2007.

World Health Organization - WHO. WHO report on the global tobacco epidemic, 2015:

Raising taxes on tobacco. 2015. Disponível em:

<http://apps.who.int/iris/bitstream/10665/178574/1/9789240694606_eng.pdf?ua=1\&ua=1>. Aceso em: 09 nov. 2017. 